\title{
When sex differences lead to extinction
}

\section{There are striking differences between the male and female forms of some species. A study of marine fossils finds that such differences come at the cost of an increased risk of extinction. SEE LETTER P.366}

\section{HANNA KOKKO}

$\mathrm{I}$ is tempting to imagine evolution as a process of endless improvement. Sexually reproducing organisms continually shuffle their genomes, trying out ever more tricks that might help the organisms to cope with the challenges they face. So why does extinction sometimes occur? Is it sheer bad luck, or can selection give rise to traits that cause a species to enter a 'danger zone' of heightened risk of extinction? On page 366, Martins et al. ${ }^{1}$ report their examination of the fossil record, and identify one such danger zone. When the males and females of a given species look substantially different, this correlates with the fossil-record presence of such species having a shorter time span than that of species in which males and females look similar.

Martins and colleagues studied fossilized ostracods, which are crustacean species of the class Ostracoda. These tiny aquatic animals look like a cross between a shrimp and a mussel. They are evolutionarily close to the former. Ostracods make up a much greater part of the fossil record than their more-famous distant cousins, the trilobites. This is good news if ostracod samples are being used to calculate estimates of extinction risk.

Moreover, thousands of ostracod species exist today. Therefore, it is known that the shape of the ostracod exoskeleton (the fossilizable part of the animal) can be used to distinguish males from females. Males are more elongated than females, because they need extra space for their reproductive organs (see Fig. 1 of ref. 1). However, in terms of overall body size, in some species the males are larger than the females, whereas in others the females are the larger sex. The scale of the differences in size and shape between males and females can range from being relatively small to being highly pronounced, depending on the species.

The authors analysed the shapes and sizes of fossil exoskeletons of 93 ostracod species. These ostracods inhabited what is now eastern Mississippi between 84 million and 66 million years ago, during the Late Cretaceous period, at a time when an interior sea split North America into eastern and western halves. The authors' analysis of fossils, along with a statistical modelling between the sexes. a given species affects its risk of extinction. approach, enabled them to uncover a curious pattern. When comparing species, it emerged that those in which males were very different from females had a poorer prognosis for continued existence. The authors' models predict a tenfold increase in extinction risk per unit time when species in which males are larger than females, with large differences in shape between the sexes, are compared with species in which the males are smaller than the females, with small differences in shape

The importance of this finding for our understanding of evolution makes it of interest to more than just ostracod enthusiasts.

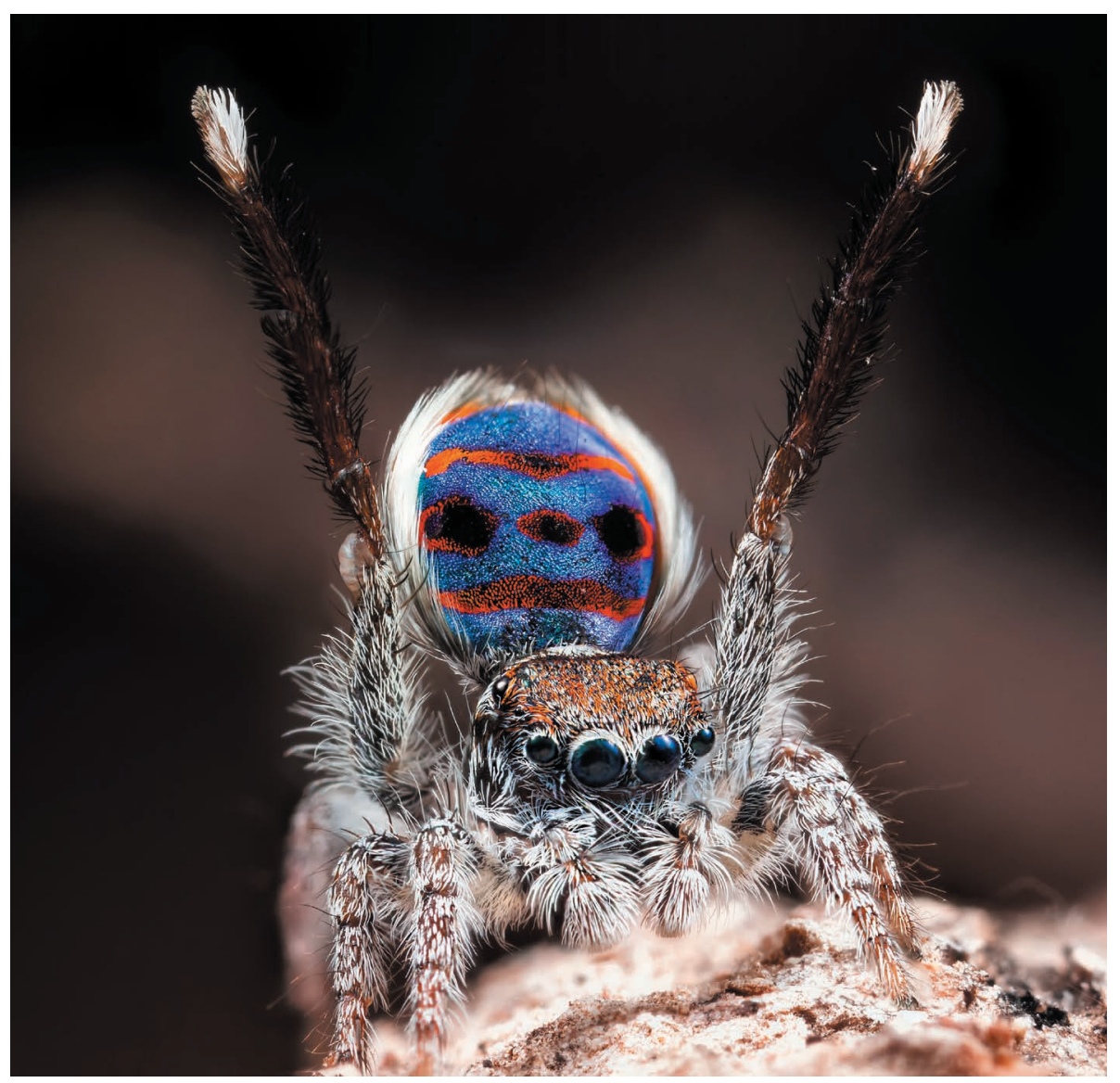

Figure 1 | The courtship dance of a male peacock spider (Maratus speciosus). Certain species have marked differences between the male and female forms - for example, male peacock spiders are
strikingly more colourful than their female counterparts. Studying fossilized aquatic creatures called marked differences between the male and female forms - for example, male peacock spiders are ostracods, Martins et al. ${ }^{1}$ investigated whether the degree of difference between male and female forms of

Sexual reproduction opens the door for sexual selection, the selection of characteristics that promote successful mating. Therefore, the generation of offspring requires both survival skills and the ability to compete for opportunities to reproduce. This can drive different selection pressures for males and females, and there is a growing appreciation in evolutionary biology that sex differences have the potential to either help or hinder the persistence of entire populations or species.

If males invest heavily in characteristics that aid different tasks from those undertaken by females, the population could benefit if strong selection weeds out suboptimally performing males and leads to the species' genome becoming better adapted over time ${ }^{2}$. However, there is also a danger that selection for male reproductive success could result in characteristics that are harmful to females ${ }^{3}$, whose ability to reproduce is more valuable for population persistence than is male input. Indeed, males of the common lizard (Lacerta vivipara) compete so intensely that their aggressive behaviour and biting of females reduces female life span and population growth ${ }^{4}$. Another simpler and perhaps under-studied effect is that, from a population-growth perspective, large, growing males consume resources that could have 
been put to better use if left for females ${ }^{5}$.

Why doesn't evolution favour asexual reproduction, to avoid the types of struggle between males and females that can have a negative effect on overall population fitness? Theoretically, an animal lineage that reproduces asexually should eventually run into difficulties. However, it was reported ${ }^{6}$ this year that Amazon molly fish (Poecilia formosa) have a genome of surprisingly good quality, even after about 500,000 generations of asexual reproduction. Many such evolutionary mysteries will provide fascinating research topics for years to come.

Martins and colleagues' approach overcomes caveats in previous attempts to measure how differences between the sexes affect population fitness. In a technique termed experimental evolution, the strength of sexual selection can be varied experimentally by restricting mating in some captive lineages to between monogamous pairs, while allowing competition for mates to operate in other lineages of the same species ${ }^{7}$. Another approach involves using information on species existing today and indicators of sexual competition, such as testis size or differences in size or colour between males and females. These indicators are then compared with the estimated risk of species extinction as documented ${ }^{8}$ in the Red List generated by the International Union for Conservation of Nature, or with the levels of population turnover ${ }^{9}$. However, experimental evolution is usually carried out in a simplified laboratory environment, whereas current threats to species persistence often have human-mediated causes. Martins and colleagues managed to show the risks of pronounced male-female differences over a long period before humans had evolved.

We can thank sexual selection for wondrous traits such as the peacock's tail, the courtship dance (Fig. 1) of the colourful male peacock spiders of the genus Maratus and, indeed, the elongated shape of male ostracods. However, as Martins et al. have shown, differences between the sexes can have negative consequences for species. With more than 10,000 ostracod species still in existence (including asexual ones), it is surprising how little we know about their genetics or other demographic factors that affect how these populations thrive, including the conditions under which they reproduce or survive well ${ }^{10}$. Why do differences between male and female ostracods result in an increase in the risk of extinction? Experimental evolution, anyone?

Hanna Kokko is in the Department of Evolutionary Biology and Environmental Studies, University of Zurich, 8057 Zurich, Switzerland.

e-mail:hanna.kokko@ieu.uzh.ch

1. Martins, M. J. F., Puckett, T. M., Lockwood, R Swaddle, J. P. \& Hunt, G. Nature 556, 366-369 (2018).

2. Whitlock, M. C. Evolution 54, 1855-1861 (2000).

3. Mank, J. E. Nature Rev. Genet. 18, 721-730 (2017)

4. Le Galliard, J.-F., Fitze, P. S., Ferrière, R. \& Clobert, J. Proc. Natl Acad. Sci. USA 102, 18231-18236 (2005).

5. Kokko, H. \& Brooks, R. Ann. Zool. Fennici 40, 207-219 (2003).

6. Warren, W. C. et al. Nature Ecol. Evol. 2, 669-679 (2018).

7. Lumley, A. J. et al. Nature 522, 470-473 (2015).

8. Morrow, E. H. \& Fricke, C. Proc. R. Soc. Lond. B 271, 2395-2401 (2004).

9. Doherty, P. F. et al. Proc. Natl Acad. Sci. USA 100, 5858-5862 (2003).

10.Park, A. W., Vandekerhove, J. \& Michalakis, Y. J. Evol. Biol. 27, 1650-1661 (2014).

This article was published online on 11 April 2018.

\section{Electronics and photonics united}

A method for integrating photonic devices with state-of-the-art nanoelectronics overcomes previous limitations. The approach shows promise for realizing high-speed, low-power optoelectronic technology. SEE LETTER P.349

\section{GORAN Z. MASHANOVICH}

$\mathrm{T}$ The integration of electronic and photonic circuits on a single silicon chip could enable unprecedented functions and performance in computing, communications and sensing at a low cost. But this goal has been hindered by the fact that most electronic circuits use bulk silicon substrates, whereas photonic circuits typically require siliconon-insulator platforms. On page 349, Atabaki et al. ${ }^{1}$ report the first fabrication of photonic devices on a bulk silicon substrate, together with millions of electronic devices known as transistors. The work paves the way for the mass production of optoelectronic systems on chips.

Photonics is prevalent in almost every aspect of day-to-day life - from smartphones and display screens to lighting and medical devices. It is often considered to be the 'electronics of the twenty-first century'. Although silicon is not an ideal photonics material (for example, lasers cannot be built from silicon), many factors have made it the main candidate for applications that require large numbers of photonic devices ${ }^{2}$. These factors include the high natural abundance of silicon, its widespread use in electronics, its optical transparency over a wide range of wavelengths and the availability of silicon-fabrication facilities that are used in micro- and nanoelectronics.

Thanks to intense research activity over the past 15 years, there have been many breakthroughs in the field of silicon photonics. Examples include hybrid silicon lasers ${ }^{3}$, various types of modulator ${ }^{4}$ (devices that convert electronic information into optical signals), high-speed light detectors ${ }^{5}$ (photodetectors) and complex optoelectronic circuits ${ }^{6}$. Several companies currently sell products based on silicon photonics chips, and many more are poised to do so in the near future.

In the electronics industry, complementarymetal-oxide-semiconductor (CMOS) technology is used to create computer processors and memory, communication chips and image sensors. This technology is based on silicon and depends on the ability to cram a large number of transistors and electronic circuits on to a single chip. Similarly, the integration of large numbers of electronic and photonic circuits on a single chip is crucial for meeting the requirements of computer processors and communication links in data centres, in terms of data-transmission rates, power consumption, scalability and complexity.

The main challenge for such integration has been the incompatibility of the material platforms used in silicon electronics and photonics. CMOS technology uses either bulk silicon substrates or thin silicon-on-insulator wafers ${ }^{6}$. The former is by far the most dominant platform because of its abundant supply chain and low cost. By contrast, silicon photonics usually requires thick silicon-on-insulator wafers that have a limited supply chain and are too expensive for many applications, such as computer memory. A long-term goal has therefore been to integrate electronic and photonic components using standard CMOSmanufacturing techniques and material platforms, without affecting the performance of such components.

Atabaki and colleagues have made a breakthrough in this regard by decoupling the formation of photonic devices from that of transistors, and by successfully incorporating these photonic devices into bulk silicon CMOS chips. The authors used standard CMOS-manufacturing methods, and introduced only a few changes to the fabrication process to create areas dedicated to photonic devices in the bulk silicon. The devices were integrated during the processing of the transistors. This involved the addition of isolated patches (islands) of the insulator material silicon dioxide to the bulk silicon and the deposition of a thin film of polycrystalline silicon on 